\title{
La professionnalisation des référenceurs aux prises avec le changement permanent
}

Jean-Claude Domenget, maître de conférences Université de Franche-Comté, Elliadd, jean-claude.domenget@univ-fcomte.fr Guillaume Sire, maître de conférences, Université Paris 2 Panthéon-Assas, Carism,

guillaume.sire@u-paris2.fr 


\title{
Résumé
}

Dans le processus de professionnalisation des référenceurs, le changement permanent imposé par Google, loin de remettre en cause la pérennité de la profession, est un facteur clé de la reconnaissance de leur fonction au sein des organisations et auprès des différents acteurs avec lesquels ils interagissent (clients, collègues, responsables, etc.). En partant du concept de professionnalisation, développé dans le champ de recherche sur la socialisation professionnelle et la construction identitaire, nous décryptons le marché du référencement en tant que système professionnel. Basé sur l'analyse croisée des résultats de deux recherches empiriques, l'une sur les référenceurs en entreprise de presse et l'autre sur les référenceurs dits "pionniers", notre raisonnement vise à expliquer pourquoi le contexte de flou et de changements permanents contribue en grande partie à la reconnaissance et à la légitimation de la profession du référenceur, et pourquoi ce même contexte peut également conduire à une remise en cause du statut des référenceurs et être mobilisé dans le discours de leurs collaborateurs comme un moyen de questionner leur crédibilité. Autrement dit, le contexte d'incertitude a cela de paradoxal qu'il contribue à rendre pérenne la profession des référenceurs tout en empêchant que le processus de professionnalisation ne s'achève.

Mots clés: professionnalisation, référenceurs, changement, légitimation, crédibilité

\begin{abstract}
If we look at the process of professionalization of SEOs, the permanent change imposed by Google does not prevent the profession to be sustainable. Actually, it is a key factor for the recognition of their function within organizations and among people with whom they interact (customers, colleagues, managers, etc.). Starting from the concept of professionalization, developed in both fields of professional socialization and identity building, we describe SEO market as a professional system. Thanks to the comparative analysis of two empirical studies, one about the role played by SEOs within the news publishing companies and the other about the identity of the SEO "pioneers", our aim was to explain why the context of vagueness and permanent changes helps largely to the recognition and legitimation of SEOs' profession, and why the same context can also lead to question the status of SEOs and their credibility. In other words, the context of uncertainty appears to be paradoxical because, on one hand, it helps making the SEOs' profession sustainable and, on the other hand, it prevents the professionalization process to be achieved.
\end{abstract}

Keywords: Professionalization, SEOs, change, legitimacy, credibility 
Le marché du référencement en France propose de nombreuses opportunités professionnelles $(37 \%$ des référenceurs ont entre 26 et 30 ans, $53 \%$ sont autodidactes, $60 \%$ ont moins de 5 ans d'expérience dans le domaine) ${ }^{10}$. En cours de professionnalisation, ce marché englobe aujourd'hui de nombreux métiers connexes ${ }^{11}$ dont l'objectif est de maximiser la visibilité sur les moteurs de recherche. En tant que système professionnel, le marché du référencement se caractérise par la présence d'un acteur hégémonique, Google, qui impose un changement permanent, avec notamment la multiplication ces dernières années des filtres de ses algorithmes et leurs mises à jour très régulières. Partant de ce contexte, plusieurs questionnements et objectifs ont été à l'origine de l'analyse dont nous rendrons compte ici : quels sont les effets des changements permanents imposés par Google sur la professionnalisation des référenceurs ? Existe-t-il des formes de permanence derrière ces changements? A quel niveau de professionnalisation cette profession se situe-t-elle? A travers ces questionnements, il s'agit de caractériser la diversité des formes de professionnalisation du marché du référencement, en partant des revendications de "professionnels» et d'apporter un regard neuf sur les relations conflictuelles entre Google et les référenceurs. Pour cela, nous partirons d'un ancrage théorique autour du concept de professionnalisation. Puis, nous présenterons la méthodologie et analyserons les résultats de deux recherches empiriques : l'une sur les référenceurs en entreprise de presse et l'autre sur les référenceurs dits «pionniers». La discussion nous permettra d'analyser les évolutions dans un contexte de flou et de changements permanents, avant d'ouvrir quelques pistes de recherches.

\section{La professionnalisation des référenceurs}

La professionnalisation est un concept bien défini, entre demande de professionnalité et exigences de professionnalisme. Adaptée au marché du référencement, elle permet de l'analyser en tant que système professionnel.

10 Données issues de l'étude emploi «Les référenceurs en France en 2014»: http://www.journaldunet.com/solutions/seo-referencement/profil-et-salaire-du-seo-francais.shtml 11 Selon les profils de référenceurs et les organisations qui les emploient, les métiers intègrent autour du référencement naturel (ou SEO pour search engine optimization) des missions liées à l'achat de liens sponsorisés, à la mesure d'audience, à la présence sur les médias socionumériques et même à la communication d'un point de vue global, le tout pouvant prendre une forme opérationnelle comme stratégique. 


\subsection{La professionnalisation entre demande de professionnalité et exigences de professionnalisme}

Dans le champ de recherche sur la socialisation professionnelle et la construction identitaire, la sociologie des groupes professionnels à la française a montré l'intérêt d'un questionnement en termes de professionnalisation. Repérée comme une tendance forte des discours et pratiques $\mathrm{du}$ monde $\mathrm{du}$ travail, «la professionnalisation serait un "processus dialectique" impliquant d'une part les travailleurs concernés, soucieux de faire reconnaître leur professionnalité, et d'autre part un ensemble d'autres acteurs (hiérarchies intermédiaires, employeurs, usagers, travailleurs concurrents, etc.) qui expriment ou imposent d'autres exigences de professionnalisme » (Dubar, Tripier et Boussard, 2011, p.316).

Il s'agit donc moins de s'intéresser à la professionnalisation, au sens de processus de reconnaissance d'un groupe professionnel, que d'analyser un phénomène plus large, celui de la montée d'une norme de professionnalisme dont la nature a changé. «Le professionnalisme n'est plus seulement la traduction d'une éthique professionnelle défendue par des travailleurs maîtrisant une expertise, il est aussi l'expression de normes organisationnelles applicables, potentiellement, à tout travailleur » (Boussard, Demazière et Milburn, 2010, p. 13).

Deux sens du terme de professionnalisation, distingués par Dubar, Tripier et Boussard, retiendront notre attention. En premier lieu, la professionnalisation concerne «l'évolution continue des compétences professionnelles d'un individu associée à une efficacité accrue » (Wittorski, 2009, p.782). Cette forme de professionnalisation renvoie au processus de fabrication par la formation, l'organisation et l'expérience d'un individu s'intégrant à un collectif de travail où il est reconnu par ses partenaires. En second lieu, la professionnalisation renvoie aux revendications de reconnaissance de soi, en tant que «professionnel », c'est-à-dire un individu à la fois qualifié, au sens de formé, et compétent, au sens de reconnu. Afin d'analyser cet « impératif de professionnalisme », ces recherches ont également montré l'importance de s'intéresser à la fois aux systèmes professionnels et aux formes identitaires des membres d'une profession, au sens de groupe professionnel (Demazière et Gardea, 2009). C'est ce que nous proposons de réaliser concernant le marché du référencement.

\subsection{Le marché du référencement en tant que système professionnel}

Analyser le marché du référencement en tant que système professionnel (Abbott, 1988) permet de souligner à la fois l'intérêt et la difficulté d'une analyse en termes de permanences et de changements. En effet, les systèmes professionnels sont porteurs de contraintes, de contradictions (des doubles injonctions comme celle pour les 
référenceurs de construire des modèles d'action, marqueurs de leur expertise alors que ceux-ci risquent d'être remis en cause à chaque changement technique), auxquelles l'individu doit se confronter, se conformer, tout en inventant sa propre façon d'être professionnel, de définir son travail et de gérer son parcours (GonninBolo, 2007).

Le système professionnel du marché du référencement se caractérise par la place hégémonique de Google qui impose un contexte de changement permanent marqué par le secret algorithmique et les effets d'annonce (recherche universelle, modifications des algorithmes, ajouts de filtres symbolisés par un bestiaire - Panda, Pingouin et Colibri - mises à jour très régulières, etc.). Un tel système peut être analysé avec le modèle social de l'« individualisme connecté»(Flichy, 2004), marqué par de nouvelles formes de travail, de nouvelles formes d'apprentissage conduisant à s'autoformer continuellement et une gestion des compétences déléguées à chaque individu. La professionnalisation des référenceurs peut en particulier être analysée à travers la tension entre autonomie et contrôle, marquant la construction d'une expertise (Dubar, Tripier et Boussard, 2011); l'importance de l'autoformation et de l'autodidaxie afin d'acquérir les compétences nécessaires, seuls gages du maintien d'une employabilité permettant aujourd'hui à la profession d'être pérenne (Domenget et Michel, 2014). Ces bouleversements des cadres du fonctionnement économique et social et des rapports que les individus entretiennent avec le travail conduisent à analyser différemment la professionnalisation.

\section{Méthodologie}

Notre analyse porte sur le croisement des résultats de deux recherches empiriques récentes et achevées. La première visait à étudier la mise en place de stratégies de référencement dans des entreprises de presse en ligne françaises. 6 référenceurs ont été interrogés en 2012/2013, ainsi que 30 acteurs travaillant en étroite collaboration avec eux. La seconde visait à étudier les trajectoires professionnelles et les pratiques de formation de référenceurs dit «pionniers», reconnus et visibles au sein de la profession. 23 entretiens ont été réalisés en 2013 auprès de professionnels qui occupaient des postes de direction, de chefs de projet au sein d'organisations (agences spécialisées ou annonceurs) ou d'indépendants. Le choix des verbatim s'est fait à partir d'une connaissance pragmatique des terrains et de la typologie des référenceurs produite précédemment (Domenget et Michel, 2014). Cinq profils de référenceurs ont été distingués, des «défricheurs» qui ont été à la source de la profession aux «stratèges » qui visent à positionner leur métier au plus haut niveau hiérarchique pour mieux exercer une mission complète de communication ou aux «planificateurs» qui ont essayé d'appliquer les techniques classiques de management pour favoriser l'évolution vers davantage de professionnalisation. Les multiples trajectoires professionnelles possibles intègrent également du côté 
« individu et dynamisme », les « explorateurs » qui défendent un esprit indépendant et une démarche opérationnelle ou du côté "collectif et statique», les « organisateurs » qui ont créé les premiers réseaux, missions et agences afin d'atteindre une pérennité du métier. Elles illustrent surtout le processus de professionnalisation (évolution constante des compétences en vue d'une efficacité accrue tout en gardant le même profil, mise en place de normes organisationnelles structurant la profession, passage d'un profil à un autre pour revendiquer une reconnaissance de soi en tant que professionnel, etc.).

Le fait d'analyser conjointement les résultats de deux recherches empiriques nous a permis de procéder à un croisement des données, selon une logique analytique en boucle (Becker, 2006) qui a permis de préciser les résultats des deux enquêtes.

\section{Les référenceurs en entreprise de presse}

La personne chargée d'améliorer le référencement des sites de presse doit composer avec des acteurs aux objectifs très différents. Bien qu'isolé au sein des entreprises de presse, ce type de référenceur fait partie du profil des « organisateurs », visant à structurer la production d'un contenu tout en intégrant les contraintes du référencement. Qui plus est, sa position est sans cesse remise en question ainsi que son professionnalisme. Le référenceur doit affirmer sa professionnalité, se justifier. Il lui faut comprendre les journalistes et leur montrer qu'il n'est pas une menace. Il doit ensuite leur faire faire certaines actions sans l'accomplissement desquelles il risquera de ne pas atteindre les objectifs fixés par ceux-là qui, eux, ne sont pas journalistes, notamment la direction et le marketing. Un processus de négociation a lieu, qui questionne sans cesse ce qu'est, ce que devrait être et ce qui est perçu de la profession du référenceur.

\subsection{Les signes de la professionnalisation}

Les principales entreprises de presse française ont embauché un référenceur à 1'interne : Le Point, L'Express, 20 minutes, Le Parisien, Le Monde, Le Figaro. Ce choix a été effectué à une étape de développement que Pablo Boczkowski nomme la «phase de rationalité » (2010, p. 48), c'est-à-dire à un moment où il ne s'agissait plus pour les entreprises de presse de montrer qu'elles étaient présentes sur le web mais également d'agir de manière à ce que leur présence soit profitable. Dès lors que des entreprises concurrentes font le même constat et procèdent au même choix coûteux de l'internalisation, c'est bien qu'il y a une reconnaissance du professionnalisme des référenceurs. Le référencement est reconnu en tant que métier et non plus seulement une mission parmi d'autres. Un poste est ouvert à cet effet et attribué à un employé qui porte le titre de référenceur plutôt qu'à un prestataire externe qualifié de « consultant chargé d'optimiser le référencement ». 
Les référenceurs rencontrés ont une vision large de leur métier :

Je suis une sorte de chef de projet transversal. [...]Je fais des recommandations techniques. Je forme les journalistes. Je fais de la veille et du testing. [...] Je les conseille. [...]Le SEO, finalement, a un rôle de manager .

$\mathrm{Au}$ sein des entreprises de presse, les professionnels du référencement revendiquent un rôle proche de l'éditorial, davantage que de la monétisation des contenus sur le marché de la publicité. Ceci justifie l'idée que les référenceurs font partie des professions de la communication. Pour eux, leur rôle est directement lié au travail des journalistes et ils aimeraient travailler à leurs côtés. « Ce serait plus logique d'être au même étage que celui où les journalistes travaillent. Parce que moi, en tout cas, je pourrais être plus utile à leur niveau, alors que la pub, je n'ai rien à faire avec eux».

Ainsi les référenceurs en entreprise de presse aspirent à « dépasser les techniques du SEO pour sortir du rapport opérationnel du référencement» (Domenget et Michel, 2014) et à être pleinement intégrés aux opérations stratégiques. Pourtant, les journalistes ont tendance à réduire leur fonction à sa seule dimension technique.

\subsection{Les relations avec les journalistes}

Quand ils travaillent pour un site d'information politique et générale, les professionnels du SEO ont conscience qu'il ne s'agit pas de maximiser la visibilité d'une information " comme les autres ». "Dans le e-commerce, je serais sûrement mieux payée, donc si je suis là, c'est d'abord pour ce qu'on fait, je veux dire ce qu'on fait ensemble, avec les journalistes, qui m'intéresse plus que de vendre des voyages $\gg$.

Dans ce cas, la maximisation de la visibilité sur Google donne aux journalistes de la crédibilité. Elle valide leur travail en les inscrivant à l'avant-scène d'un espace public dont la particularité est que certains propos y sont «plus publics que d'autres » (Cardon, 2010). Plusieurs normes de professionnalisme se mettent en place, à la fois conjointes et conflictuelles, entre journalistes et référenceurs. En effet, dans un univers où leur production côtoie celles des communicants et des amateurs, les journalistes cherchent à gagner en visibilité dans le but d'être des leaders d'opinion: «écrire pour être lus » (Siméant, 1992, p. 40). Or, puisque Google est un des principaux, sinon le principal, régisseurs de l'échelle de visibilité du web, la mise en lumière de la production journalistique par ces services pourrait constituer un moyen de «labelliser l'information-presse par rapport à toutes les autres » (Wolton, 2003, p. 14). C'est pourquoi le référenceur n'est pas forcément une gêne pour les journalistes, dans le sens où son action serait liée aux seuls impératifs économiques, mais peut au contraire constituer un atout pour des 
journalistes cherchant eux-mêmes à devenir légitimes sur le web au même titre qu'ils le sont sur les autres médias. Pourtant, il peut aussi être perçu comme un frein, justement parce que les contours de la profession des webjournalistes ne sont pas stabilisés, et que ceux-là sont par conséquent jaloux de certaines prérogatives dont ils estiment que les autres professions n'ont pas à être dotées.

Dans toutes les entreprises de presse où il y a un référenceur, celui-ci interagit avec les journalistes de manière formelle lors des conférences de rédaction ou à l'occasion de formations organisées régulièrement, et de manière informelle, au jour le jour, pour répondre à leurs questions.

J'essaie d'aller aux conférences de rédaction hebdomadaires. Pourquoi ? Pour leur donner des pistes, des sujets. J'ai vu que tel ou tel mot-clé était passé dans l'actu, est-ce que vous l'avez vu ? J'essaie de donner des sujets sans l'imposer [...], des petites choses qui sont recherchées par les internautes et que les journalistes ne vont pas forcément avoir en tête.

Quant aux journalistes, s'ils reconnaissent un certain professionnalisme au référenceur, ce dernier doit pour autant rester à sa place et ne pas avoir d'ascendant hiérarchique à leur endroit.

Je ne supporterais pas que [le référenceur] ait le pouvoir de me dire ce que je dois faire ou non, quand, et qu'il soit une espèce de deuxième rédac' chef. Il n'est quand même pas journaliste. Donc je veux bien qu'il me donne des conseils, mais pas des ordres. Il est hors de question qu'il me donne des ordres.

Les journalistes acceptent de collaborer avec une profession qui n'est pas la leur mais jusqu'à un certain point, définissant ainsi de leur point de vue un territoire qui de facto ne pourrait pas appartenir au champ du référenceur. Lorsque ce dernier intervient dans la politique éditoriale, il est donc nécessaire de ne pas proposer un sujet dont le seul intérêt serait lié à sa capacité à se hisser en haut des classements de Google. Cela rejoint les observations effectuées par Murray Dick dans la rédaction du Guardian : « [Le référenceur] est conscient qu'en proposant des sujets jugés non pertinents, il pourrait cesser d'intéresser ses partenaires et donc perdre du pouvoir au sein de l'organisation » (2011, p. 472). Le profil du référenceur est donc marqué par l'incertitude.

\subsection{Un profil marqué par l'incertitude}

L'incertitude quant aux actions préconisées par le référenceur et aux résultats qu'elles sont censées provoquer contribue, d'une part, à assurer la reconnaissance du professionnalisme du référenceur, parce que les autres professions ne comprennent pas ce qu'il fait; d'autre part, et paradoxalement, cela peut aussi menacer cette reconnaissance, puisqu'il peut lui être reproché de ne servir à « rien », et d'être dans 
l'incapacité de prouver rapidement, objectivement, évidemment, qu'il sert à « quelque chose ». Il est en effet très difficile pour le référenceur de montrer ce que ses actions ont apporté, dès lors que ses actions consistent essentiellement à influencer les actions des journalistes, des équipes marketing et des équipes techniques.

Le flou nimbant le fonctionnement exact du moteur de recherche de Google rend nécessaire la veille effectuée par le référenceur à ce sujet, dont la pratique devient ainsi constitutive de sa légitimité dans l'entreprise. Certains référenceurs entretiennent des relations directes avec les employés de Google, à qui ils téléphonent régulièrement, ce qui peut également contribuer à asseoir leur légitimité. Un sentiment de dépendance se crée, non plus envers Google directement, mais envers l'employé chargé d'optimiser le référencement des contenus sur Google et procure au référenceur une certaine autonomie.

L'autonomie qui est une des caractéristiques de leur métier est appréciée par les référenceurs en entreprises de presse. Elle leur offre une possibilité de déplacement, comme des électrons libres, entre les services techniques, le marketing et la rédaction. L'absence d'objectifs chiffrés en termes de trafic leur permet d'évacuer une certaine anxiété que pourrait provoquer l'obligation de résultat. Parce qu'ils ont une « compétence particulière » et une «spécialisation fonctionnelle 》 (Crozier et Friedberg, 1977, p. 83) uniques, ils jouissent d'une forme de pouvoir dans l'organisation, qui n'est jamais autoritaire ou incontournable, mais qui est bien réelle. «[Le SEO], c'est très flou pour tout le monde, et pour moi aussi parfois, mais pour moi c'est quand même moins flou. Et c'est grâce à ce flou que, dans la boîte, j'ai mon indépendance et ma marge d'action ».

Néanmoins, l'autre versant de cette autonomie dans l'organisation est un risque d'isolement. L'isolement des référenceurs dans les entreprises de presse participe alors à la difficulté de faire reconnaitre leur professionnalisme. Il n'y a en effet le plus souvent qu'un seul référenceur par entreprise, et celui-ci ne se sent véritablement proche d'aucune autre profession, soit parce qu'il ne veut pas s'en rapprocher (comme c'est parfois le cas avec les services du marketing), soit parce que ce sont ces professions qui ne veulent pas se rapprocher de lui (comme c'est parfois le cas avec les journalistes).

De même, l'incertitude quant aux résultats de ses actions conduit certains acteurs à penser que, dès lors que l'algorithme de Google est inconnu et qu'il change régulièrement, cela ne sert à rien d'embaucher un référenceur, car la partie est perdue d'avance ; autant se concentrer sur ce qu'on sait faire : produire du contenu.

\section{Les référenceurs dits " pionniers »}

Nous élargissons à présent notre focale analytique pour nous intéresser non plus aux seuls référenceurs des contenus de presse mais aux référenceurs au sens large, 
dont l'activité est consacrée à des contenus de types très différents, et dont la particularité réside donc avant tout dans le fait que leur métier à tous consiste à essayer d'augmenter l'apport de trafic en provenance de Google.

\subsection{Permanences dans la professionnalisation}

La professionnalisation du référencement repose sur des formes permanentes du métier. Un noyau invariant de compétences et le rôle continu de la formation auprès des pairs a donné naissance à une communauté SEO.

Faire du référencement consiste à intégrer un ensemble de pratiques, de codes, de conventions, de connaissances, d'aptitudes, de compétences, de savoir-faire voire de comportements, de cultures propres aux métiers de référenceur. «En agence, il faut avoir des bases, être un bon SEO, avoir développé des stratégies sur des sites différents mais ça ne suffit pas. La dimension relationnelle est essentielle, notamment avec le client $\gg$.

Le rôle continu de la formation auprès des pairs a donné naissance à une communauté d'intérêt (Proulx, 2006), dans laquelle se sont développés un sentiment et des règles d'appartenance participant à la création d'une identité collective. Ce qui a fait lien dès le début, c'est ce besoin d'échanges, de partage des tests afin de monter en compétences individuellement par le collectif. Aujourd'hui, l'atmosphère beaucoup plus pacifiée, d'un avis général, traduit une maturité de la profession et une marche vers la reconnaissance de professionnalisme du métier. L'autoformation auprès des pairs reste un pilier de la professionnalisation. Il semble en effet indispensable pour tous de «se considérer en formation permanente 》 dans une profession en changement constant.

Avec une grille de lecture en termes de permanences et changements, un paradoxe apparaît autour d'une idée de continuité dans la volatilité du marché. En effet, le marché du référencement a toujours été volatile. «Le problème fondamental reste la rétention des clients ». Qu'ils soient indépendants et jouent principalement sur leur réputation ou une agence qui porte en bannière son client historique, le «turn over » de clients est important. Car à la différence des liens sponsorisés avec lesquels la mesure du retour sur investissement est immédiate, le talon d'Achille du SEO reste l'absence de critères de mesure d'efficacité fiables. Les interviewés ont très bien expliqué la part de feeling, d'expérience, de tests qui persiste dans le métier, même si en fonction des typologies de sites des problématiques récurrentes se font jour. Le référencement reste l'affaire d'une «alchimie » qui entretient une part de magie du métier, difficilement conciliable avec les standards d'efficacité marketing attendus aujourd'hui par le marché.

Cette volatilité du marché donne cette fragilité qui pousse à la professionnalisation et assure à cette profession une évolutivité suffisante afin d'absorber les innovations, petites ou grandes. 


\subsection{Changements au cours de la professionnalisation}

Dans le premier sens du terme, la professionnalisation du référencement a été marquée par cette évolution continue des compétences professionnelles associée à une efficacité accrue, définie par Wittorski (2009). Du côté des profils qui sont dans l'évolution du métier, les « explorateurs » sont par définition dans la recherche de la performance maximale. Leurs compétences sont de plus en plus accrues qu'ils soient dans le domaine technique, éditorial ou de la création de liens, avec la mise au point de techniques propres (PR sculpting il y a quelques années, linkninja, référencement opportuniste... la liste s'allonge régulièrement...). L'arrivée progressive dans la profession de «stratèges » illustre à son tour l'évolution des compétences, quittant l'aspect opérationnel pour remplir des missions de plus en plus stratégiques et globales. Du côté des profils qui sont dans la consolidation de la profession, les « organisateurs » ont créé par exemple les premières agences avec souvent des outils de reporting maison, avant que les «planificateurs» ont cherché à sortir du one shot, marqué par une tarification trop basée sur la performance, pour aller vers un accompagnement inspiré par les grands cabinets d'audit. On peut considérer que leur action est déjà préstratégique car elle reconnaît la diversité des missions et a proposé dès le début de modéliser les structures clientes, de segmenter les offres et de trouver des modèles économiques pérennes. Quel que soit le profil de référenceur pris en compte, les évolutions de leurs compétences sont marquées par une recherche d'efficacité accrue. "On a essuyé les plâtres, évangélisé le marché. Au début, il fallait expliquer ce qu'était un moteur de recherche, convaincre les gens de faire du référencement. Plus maintenant. [...] On a construit l'écosystème, les méthodes, les prestations, les outils créés par les SEO».

Dans le deuxième sens, la professionnalisation renvoie aux revendications de reconnaissance de soi, en tant que « professionnel », c'est-à-dire un individu à la fois qualifié, au sens de formé, et compétent, au sens de reconnu, ainsi qu'aux exigences de professionnalisme qui sont accrues de la part de leurs partenaires et clients. L'injonction au professionnalisme (Boussard, Demazière et Milburn, 2010) des référenceurs conduit à vouloir mesurer chacune des actions préconisées. Ces exigences de professionnalisme font évoluer les missions beaucoup plus qu'une dépendance aux modifications des algorithmes de Google et aux derniers filtres apparus. "Quelles compétences avoir en 2013? Le pilotage mais c'est très compliqué. Partir des outils de Web analyse, c'est très différent des prestations de mots-clés ».

La dynamique des trajectoires professionnelles et les opportunités professionnelles illustrent enfin les changements au cours de la professionnalisation. Plutôt que de vouloir rechercher des parcours-types de formation et d'évolutions de carrière, le marché du référencement est marqué par une grande dynamique des trajectoires professionnelles (Domenget et Michel, 2014). Il n'existe évidemment pas une 
trajectoire professionnelle unique, allant de l'opérationnel («avoir la main dans le cambouis ») - coder, produire du contenu, partir à la pêche aux liens — à la définition de la stratégie SEO ou même de la stratégie de communication de grands comptes. Tous les pionniers ne sont pas devenus des stratèges. Certains veulent absolument rester dans leur «cœur de métier», d'autres ont quitté le SEO et sont partis dans des stratégies transversales. Il est tout autant possible de faire le chemin inverse et de passer d'une fonction stratégique à un retour à l'opérationnel.

\subsection{Une relation ambivalente à Google}

Quant à la relation avec Google, elle reste ambivalente malgré une forme de reconnaissance de la profession par le moteur ${ }^{12}$. Ils sont à la fois «Google dépendants » et ont "plus de connaissance sur le fonctionnement du moteur que les équipes en interne ». Comme nous l'avions déjà analysé, cette relation ambivalente a peut-être fondé définitivement la pérennité du métier et conduit à la nouvelle étape de professionnalisation, la quête de légitimité (Domenget et Michel, 2014). Elle se base sur les changements permanents imposés par Google. «Avec Google, on est un peu comme sur des sables mouvants, avec Panda et Pingouin. On ne sait comment le site va réagir. Je suis toujours très prudent sur les résultats escomptés ».

Certes, les référenceurs étaient prêts : leurs pratiques, leurs astuces, leurs forums, les avaient conduits à un niveau d'expertise et de reconnaissance de leurs compétences leur permettant de se situer favorablement par rapport à leurs clients. Mais c'est surtout leur capacité, on pourrait dire à « digérer » toutes les innovations, qui a apporté la preuve de leur professionnalisme, aussi bien en interne qu'en externe. Les profils-types distingués montrent comment ils y sont parvenus, tous avec des recettes différentes, de l'« explorateur» au «planificateur ».

\section{Discussion autour du flou et des changements permanents}

Le contexte de flou, de changements permanents et d'interdépendance au sein du marché du référencement contribue en grande partie à la reconnaissance du professionnalisme du référenceur. Les référenceurs l'utilisent pour tenir un discours

12 Le 13 mars 2014 a été lancé le «blog officiel pour les webmasters » qui se propose d'être un espace d'échanges avec la «communauté des webmasters français » et représente surtout une évolution importante dans les relations longtemps conflictuelles entre le moteur et les référenceurs. http://googlewebmastercentral-fr.blogspot.fr/ 
de crédibilité. Pourtant, ce contexte d'incertitude explique également pourquoi le processus de professionnalisation des référenceurs reste inachevé.

\subsection{La reconnaissance du professionnalisme du référenceur}

L'analyse conjointe des deux terrains de recherche proposée dans cet article montre que le flou et les changements permanents participent d'une reconnaissance $\mathrm{du}$ groupe professionnel des référenceurs. La reconnaissance de ce groupe professionnel (Demazière, Gadéa, 2009) passe notamment par des actions de cadrage et de contrôle du travail de ces professionnels dans les organisations au sein desquelles ils sont employés. Des formes de normalisation des activités du référenceur se sont progressivement mises en place, sous la double action d'un travail de régulation par les référenceurs eux-mêmes du flou et du changement permanent entretenu par cet acteur hégémonique du système qu'est Google.

Il nous semble intéressant ici de considérer que le référenceur joue le rôle d'un « centre de traduction», comme cela est défini par la théorie de l'acteur-réseau (Akrich et al., 2006). Un centre de traduction, c'est un acteur, engagé dans un collectif sociotechnique agissant, capable de mobiliser l'ensemble de ce collectif afin d'engager certaines actions (Callon, 2006, p. 270). Le référenceur correspond à cette description étant donné son rôle d'interface: il traduit les règles du référencement sur Google dans une langage et une "grammaire d'action» (Lemieux, 2009) acceptable pour les journalistes. En retour, il traduit la production journalistique dans un langage compréhensif pour Google en ajoutant des balises méta-textuelles, des liens hypertextes, des sitemaps visant à aider le moteur de recherche à comprendre de quoi il est question dans les documents et quelle est leur pertinence au regard de l'actualité et des requêtes effectuées sur son moteur. De même, le référenceur est également un centre de traduction à l'intérieur de l'entreprise de presse, où il essaie de concilier les objectifs des journalistes et les siens avec ceux des équipes commerciales et des équipes techniques. S'il ne réussit pas à rallier les autres à sa cause, alors son action échouera, car il a besoin que les équipes techniques, commerciales et rédactionnelles entreprennent des actions qu'il ne peut pas entreprendre à leur place.

\subsection{Un discours de crédibilité}

Un deuxième sujet de discussion porte sur la forme de permanence dans les discours des différents acteurs du système professionnel du référencement. En effet, le discours de l'acteur dominant (Google) est utilisé par les acteurs intermédiaires (les référenceurs) pour entretenir cette idée de changement permanent auprès d'un troisième acteur (leurs clients) et faire ainsi reconnaître leur professionnalité, leur expertise. Le discours de Google est celui d'une amélioration permanente de 
l'algorithme vers plus de pertinence (Cardon, 2013). Chaque année, la sempiternelle question des (r)évolutions à digérer est l'objet des conseils de début d'année de nombreux blogs spécialisés et occupe une place importante des thématiques du SEO Campus ${ }^{13}$. Pourtant, lorsque ces professionnels sont questionnés sur ce qui constitue leur expertise, les réponses sont invariantes. «Les fondamentaux restent les mêmes ». "Une alchimie du SEO » est à doser en fonction beaucoup plus des types de sites que des changements de Google. Ces formes de permanences dans les discours des référenceurs montrent qu'il s'agit d'un discours pour rendre crédible une expertise (Garric, Léglise, 2012). Pour les référenceurs, l'enjeu principal est de persuader leurs clients qu'ils ont le pouvoir de le faire, qu'ils ont la capacité de digérer les changements permanents.

\subsection{Un processus de professionnalisation inachevé}

En guise de troisième et dernier sujet de discussion, il semblerait paradoxalement que ce soit le contexte de flou et de changement qui empêche le processus de professionnalisation de "s'achever », en cela notamment qu'il crée une incertitude quant à l'efficacité de l'action du référenceur. Ce dernier ne peut en effet jamais prouver quelles ont été les retombées de son travail. Il ne peut pas non plus assurer que sans lui le trafic capté sur Google n'aurait pas été le même. Cela peut rendre difficile le renouvellement des contrats, notamment dans un contexte de crise économique. Le référenceur doit sans cesse persuader (il ne peut pas convaincre ou démontrer) que son action est nécessaire. Par ailleurs, le flou et le changement créent une situation où son action peut être cantonnée à la technique, justement parce qu'on n'est pas sûr (et qu'on ne peut pas prouver) que ce soit « utile » d'adapter l'éditorial à la stratégie de référencement. Or, nous avons vu que les référenceurs considéraient que l'aspect multiple de leur activité était constitutif de leur profession, et que celleci ne pouvait pas être réduite à sa seule dimension technique.

De même, la professionnalisation du référenceur peut se heurter à d'autres processus de professionnalisation, qui eux-aussi sont encore jeunes, encore préoccupés par les questions de légitimité. Le web est en effet un écosystème qui n'a qu'un peu plus de vingt ans. Le dynamisme de ses mutations freine la stabilisation de certaines professions. C'est notamment le cas des journalistes, à la recherche d'une légitimité sur le web. Ils peuvent voir d'un mauvais œil le fait que le référenceur se mêle de ce qu'ils considèrent être leurs prérogatives. Dans ce genre de situation, l'autonomie et l'indépendance des référenceurs peuvent jouer en leur défaveur en les

13 Voir les programmes des SEO Campus, "L'événement Search Marketing », qui proposent d'analyser les changements chez Google. http://seo-campus.org/ 
privant d'alliés au sein de l'entreprise, tandis que les journalistes pourraient plus facilement « faire bloc ».

\section{Conclusion}

Dans cet article, nous avons vu pourquoi le contexte d'incertitude dans lequel évoluent les référenceurs a cela de paradoxal qu'il est à la fois ce qui contribue à rendre pérenne la profession des référenceurs tout en empêchant que le processus de professionnalisation ne s'achève. Il nous semble qu'un tel paradoxe est assez caractéristique d'évolutions que l'on peut observer aujourd'hui dans le secteur de la communication. Certains professionnels - comme les référenceurs, les community managers, les webmasters, les développeurs d'applications - doivent composer avec des technologies dont ils n'ont pas les moyens de connaître le fonctionnement dans le détail et dont la plupart appartiennent à des multinationales américaines avec lesquelles il est difficile d'avoir une relation étroite. Ces professionnels se déplacent et agissent dans un contexte flou, où leur compétence est moins de dissiper le brouillard ou de voir au travers que de savoir s'en débrouiller. Ils développent une expérience qui tient autant à l'instinct qu'à la compétence et à l'expérience.

Ces professionnels de la communication se retrouvent dans une situation où l'un de leurs partenaires, de leurs collègues, de leurs clients, de leurs employeurs peut à tout moment leur demander : « finalement, à quoi sers-tu? ». Que répondre ? Sinon, ne plus rien faire de façon à ce que le sceptique soit forcé de constater empiriquement que le métier pointé, contrairement à ce qu'il croyait, est bel et bien utile et même indispensable.

Une telle situation pose des questions en termes de place dans l'organisation, de niveau de salaire et de formation. Il est en effet difficile de savoir comment positionner spatialement et hiérarchiquement ces professionnels dans l'entreprise. Il est difficile également de savoir comment les rémunérer, puisqu'on ne peut jamais mesurer sans ambiguité leur apport à la création de valeur ajoutée. Il est difficile encore de savoir comment il faut les former, puisque l'instinct est une qualité qui ne s'acquiert que par l'expérience, mais qui peut très bien ne jamais être acquise même après une longue expérience. Enfin, il se trouve que, pour ajouter du brouillard au brouillard, les compétences nécessaires pour de tels métiers changent souvent sans qu'on ne soit jamais sûr qu'elles ont effectivement changé.

\section{Bibliographie}

Abbott, A. (1988). The System of the Professions. An Essay of the Division of Expert Labour. Chicago : Chicago University Press. 
40 Dynamiques de professionnalisation en communication

Akrich M., Callon M. et Latour B. (2006). Sociologie de la traduction. Textes fondateurs. Paris : Presses des Mines.

Becker, H. S. (2006). Le Travail sociologique. Méthode et substance. Fribourg: Academic Press Fribourg - Ed. Saint Paul.

Boczkowski, P. J. (2010). Ethnographie d'une rédaction en ligne argentine. Les logiques contraires de la production de l'information chaude et froide. Réseaux, (160-161), 43-78.

Boussard, V., Demazière, D. et Milburn, Ph. (2010). L'injonction au professionnalisme. Analyses d'une dynamique plurielle. Rennes: Presses Universitaires de Rennes.

Callon, M. (2006), Sociologie de l'acteur réseau. Dans M. Akrich, M. Callon M. et B. Latour. Sociologie de la traduction. Textes fondateurs (p. 267-276). Paris : Presses des Mines.

Cardon, D. (2010). La démocratie Internet. Promesses et limites. Paris : Seuil.

Cardon, D. (2013). Dans l'esprit du PageRank. Une enquête dans l'algorithme de Google. Réseaux, (177), 63-95.

Crozier, M. et Friedberg, E. (1977). L'acteur et le système. Les contraintes de l'action collective. Paris : Seuil.

Demazière, D. et Gadéa, C. (2009). Sociologie des groupes professionnels, Acquis récents et nouveaux défis. Paris : La Découverte.

Dick, M. (2011). Search engine optimisation in UK news production, Journalism Practice, 5(4), 462-477.

Domenget, J-C. et Michel, J-L. (2014). Le métier de référenceur est-il pérenne et légitime en communication? Dans V. Lépine et M. D. David (coord.). Entre réflexif et prescriptif : analyse des dispositifs d'apprentissage et de formation des communicateurs. Les Cahiers du Resiproc, (2), 161-179.

Dubar, C., Tripier, P. et Boussard, V. (2011). Sociologie des professions ( $3^{\mathrm{e}}$ éd.). Paris : Armand Colin.

Flichy, P. (2004). L'individualisme connecté entre la technique numérique et la société. Réseaux, (124), 17-51.

Garric, N. et Léglise, I. (2012). Analyser le discours d'expert et d'expertise. Dans I. Léglise et N. Garric (dir.), Discours d'expert et d'expertise (p. 1-16). Berne : Peter Lang.

Gonnin-Bolo, A. (dir.) (2007). Parcours professionnels. Des métiers pour autrui : entre contraintes et plaisir. Paris : Belin. 
Lemieux C. (2009). Le devoir et la grâce. Paris : Economica.

Siméant, J. (1992). Déontologie et crédibilité. Le réglage des relations professionnelles au CFJ. Politix, 5(19), 37-55.

Wolton, D. (2003). Journalistes, une si fragile victoire... Hermès, (35), 9-21.

Wittorski, R. (2009). A propos de la professionnalisation. Dans J-M Barbier, E. Bourgeois et G. Chapelle (dir.). Encyclopédie de l'éducation et de la formation (p. 781-793). Paris : PUF. 